\title{
Dispersion relations and speeds of sound in special sectors for the integrable chain with alternating spins
}

\author{
B - D Dörfel用 and St Meißner£ \\ Institut für Physik, Humboldt-Universität, Theorie der Elementarteilchen \\ Invalidenstraße 110, 10115 Berlin, Germany
}

\begin{abstract}
Based on our previous analysis [1] of the anisotropic integrable chain consisting of spins $s=\frac{1}{2}$ and $s=1$ we compare the dispersion relations for the sectors with infinite Fermi zones. Further we calculate the speeds of sound for regions close to sector borders, where the Fermi radii either vanish or diverge, and compare the results.
\end{abstract}

PACS numbers: 75.10 JM, 75.40 Fa

$\ddagger$ E-mail: doerfel@qft2.physik.hu-berlin.de

$\S$ E-mail: meissner@qft2.physik.hu-berlin.de 


\section{Introduction}

The integrable spin chain $X X Z\left(\frac{1}{2}, 1\right)$ constructed in 1992 by de Vega and Woynarovich [2] shows a rich physical structure with different ground states depending on the anisotropy parameter and the two coupling constants. In our previous papers [1], [3], [4] and [5] we have analyzed this structure and calculated several important quantities. In this paper we deal mainly with dispersion relations and speeds of sound.

The paper is organized as follows. After having reviewed the definitions in section 2 we found it necessary to remind the reader results of our last paper [1] concerning the phase diagram. In the same section the dispersion relations for all sectors with infinite Fermi zones only are compared. In section 4 we present the calculations for the speeds of sound obtained either by power expansion or Wiener-Hopf technique for small or large Fermi radii respectively.

Our conclusions are contained in section 5. Some useful definitions are compiled in an appendix.

\section{Description of the model}

We refer the reader to papers [2] and [3] for the basics of the model.

Our Hamiltonian of a spin chain of length $2 N$ is given by

$$
\mathcal{H}(\gamma)=\bar{c} \overline{\mathcal{H}}(\gamma)+\tilde{c} \tilde{\mathcal{H}}(\gamma)
$$

with the two couplings $\tilde{c}$ and $\bar{c}$. The anisotropy parameter $\gamma$ is limited to $0<\gamma<\pi / 2$. For convenience we repeat the Bethe ansatz equations (BAE), the magnon energies and momenta and the spin projection.

$$
\begin{aligned}
\left(\frac{\sinh \left(\lambda_{j}+i \frac{\gamma}{2}\right)}{\sinh \left(\lambda_{j}-i \frac{\gamma}{2}\right)} \frac{\sinh \left(\lambda_{j}+i \gamma\right)}{\sinh \left(\lambda_{j}-i \gamma\right)}\right)^{N}=-\prod_{k=1}^{M} \frac{\sinh \left(\lambda_{j}-\lambda_{k}+i \gamma\right)}{\sinh \left(\lambda_{j}-\lambda_{k}-i \gamma\right)}, \quad j=1 \ldots M, \\
E=\bar{c} \bar{E}+\tilde{c} \tilde{E}, \\
\bar{E}=-\sum_{j=1}^{M} \frac{2 \sin \gamma}{\cosh 2 \lambda_{j}-\cos \gamma}, \\
\tilde{E}=-\sum_{j=1}^{M} \frac{2 \sin 2 \gamma}{\cosh 2 \lambda_{j}-\cos 2 \gamma}, \\
P=\frac{i}{2} \sum_{j=1}^{M}\left\{\log \left(\frac{\sinh \left(\lambda_{j}+i \frac{\gamma}{2}\right)}{\sinh \left(\lambda_{j}-i \frac{\gamma}{2}\right)}\right)+\log \left(\frac{\sinh \left(\lambda_{j}+i \gamma\right)}{\sinh \left(\lambda_{j}-i \gamma\right)}\right)\right\}, \\
S_{z}=\frac{3 N}{2}-M .
\end{aligned}
$$




\begin{tabular}{|c||c|}
\hline 0 & $(1,+)^{\infty},(2,+)^{\infty}$ \\
\hline I & $(1,-)^{\infty},(1,+)^{f, 0}$ \\
\hline II & $(1,-)^{\infty},(1,+)^{\infty}$ \\
\hline III & $(1,-)^{\infty},(1,+)^{f, \infty}$ \\
\hline IV & $(1,-)^{\infty}$ \\
\hline V & $(1,-)^{\infty},(2,+)^{f, 0}$ \\
\hline VI & $(1,-)^{f, \infty},(2,+)^{\infty}$ \\
\hline
\end{tabular}

Table 1. All sectors appearing in the phase diagram. Upper indices indicate infinite and finite Fermi zones. In the latter case, the second index distinguishes, wether the filling starts at $\lambda=0$ or $\lambda=\infty$.

\section{Phase structure and dispersion relations}

In section 4 of [可] we have carried out the complete analysis of the ground state structure based on the solution of the thermodynamic Bethe ansatz. We will now review the main results.

Using different sets of special anisotropy points we were able to prove that (for $0<\gamma<\pi / 2$ ) only three kinds of strings occur in the ground state. The picture changes remarkably at $\gamma=\pi / 3$ and $\gamma=2 \pi / 5$. All possible sectors are compiled in table 1 taken from [1]. To keep our notation consistent we found it useful to call the sector already discussed in [2] sector 0 . In the $(\tilde{c}, \bar{c})$-plane the sector borders are straight lines starting from the origin. Moving counterclockwise from the $\tilde{c}$-axis the sectors follow in sequence 0 , I, IV, V if $0<\gamma \leq \pi / 3$. For $\pi / 3<\gamma<2 \pi / 5$ the sequence is 0 , II, I, IV, VI and for $2 \pi / 5<\gamma<\pi / 2$ one has 0 , II, III, IV, VI. In case $\gamma=2 \pi / 5$ sector II is followed by IV and neither I or III occur. For details see figure 1 in [1].

To compare the dispersion relations in the sectors 0 , II and IV it is convenient to work with the functions

$$
p(\lambda, \alpha, \beta)=\arctan \frac{\sinh (\pi \lambda / \beta)}{\cos (\pi \alpha / 2 \beta)}
$$

and

$$
g(\lambda, \alpha, \beta)=\frac{4 \pi}{\beta} \frac{\cos (\pi \alpha / 2 \beta) \cosh (\pi \lambda / \beta)}{\cosh (2 \pi \lambda / \beta)+\cos (\pi \alpha / 2 \beta)} .
$$

While $p$ is odd, $g$ is an even function of $\lambda$. Both are connected by

$$
\frac{d}{d \lambda} p(\lambda, \alpha, \beta)=\frac{1}{2} g(\lambda, \alpha, \beta) \text {. }
$$

By straightforward calculation one may prove the relation

$$
\sin \left(\frac{\pi}{2}+p(\lambda, \alpha, \beta)\right) \sqrt{\cos ^{2} p(\lambda, \alpha, \beta)+\frac{\sin ^{2} p(\lambda, \alpha, \beta)}{\cos ^{2}(\pi \alpha / 2 \beta)}}=\frac{\beta}{2 \pi} g(\lambda, \alpha, \beta) .
$$

We remark that in [4] we used a function $g(\lambda, \alpha)$ related by

$$
g(\lambda, \alpha, \pi-\gamma)=g(\lambda, \alpha)
$$


Let us first consider sector 0 . The results are given in [2] taking into account our definition of momentum in equation (2.4). One has two elementary physical excitations, the holes in the distributions of the $(1,+)$ - and $(2,+)$-strings respectively. We label them by index $i, i=1,2$.

$$
\begin{aligned}
& p_{h}^{i}\left(\lambda=\lambda_{h}^{i}\right)=\frac{\pi}{4}+\frac{1}{2} p(\lambda, 0, \gamma), \\
& \varepsilon_{h}^{i}(\lambda)=c^{i} \frac{1}{2} g(\lambda, 0, \gamma)
\end{aligned}
$$

with $c^{1}=\bar{c}$ and $c^{2}=\tilde{c}$.

From equation (3.4) the dispersion relations follow

$$
\varepsilon_{h}^{i}\left(p_{h}^{i}\right)=\frac{2 \pi}{\gamma} c^{i} \frac{\sin 2 p_{h}^{i}}{2} .
$$

One may say that the dispersion relations diagonalize in the coupling constants. This fact changes in the other two sectors.

Next we look at sector IV which contains the case of negative couplings. The exact border lines are given in [5]. The elementary physical excitations are holes in the distribution of the $(1,-)$-strings. We quote the result from our paper [3], equations (4.5) and (4.6)

$$
\begin{aligned}
& p_{h}(\lambda)=\frac{3 \pi}{2}+\frac{1}{2} p(\lambda, 0, \pi-\gamma)+p(\lambda, \gamma, \pi-\gamma), \\
& \varepsilon_{h}(\lambda)=-\frac{\bar{c}}{2} g(\lambda, 0, \pi-\gamma)-\tilde{c} g(\lambda, \gamma, \pi-\gamma) .
\end{aligned}
$$

The dispersion relation cannot be obtained in a closed form. One has to invert the monotone function (3.9) and to substitute it in equation (3.10). Numerical calculations show an sine-like behaviour, see the broken lines in figure 2 of [3].

In [1] we found the new sector II, existing only for $\gamma>\pi / 3$ (for the exact border lines see next section). Here the excitations are $(1,+)$ - and $(1,-)$-string holes. The dispersion law for the $(1,-)$-string holes is simple:

$$
\begin{aligned}
& p_{h}^{1-}(\lambda)=\frac{\pi}{4}+\frac{1}{2} p(\lambda, 0, \pi-2 \gamma), \\
& \varepsilon_{h}^{1-}(\lambda)=-\frac{\tilde{c}}{2} g(\lambda, 0, \pi-2 \gamma), \\
& \varepsilon_{h}^{1-}\left(p_{h}^{1-}\right)=-\frac{2 \pi \tilde{c}}{\pi-2 \gamma} \frac{\sin 2 p_{h}^{1-}}{2} .
\end{aligned}
$$

To write down the case of $(1,+)$-strings it is necessary to introduce a new function $d_{1}(\lambda)$

$$
d_{1}(\lambda)=\frac{1}{2 \pi} \int_{-\infty}^{\infty} e^{i \omega \lambda} \frac{\cosh (\omega(\pi-3 \gamma) / 2) d \omega}{2 \cosh (\omega(\pi-2 \gamma) / 2) \cosh (\omega \gamma / 2)}
$$

already used in [1]. We further define another function $e_{1}(\lambda)$ by

$$
\frac{d}{d \lambda} e_{1}(\lambda)=d_{1}(\lambda) \quad \text { and } \quad e_{1}(0)=0 .
$$




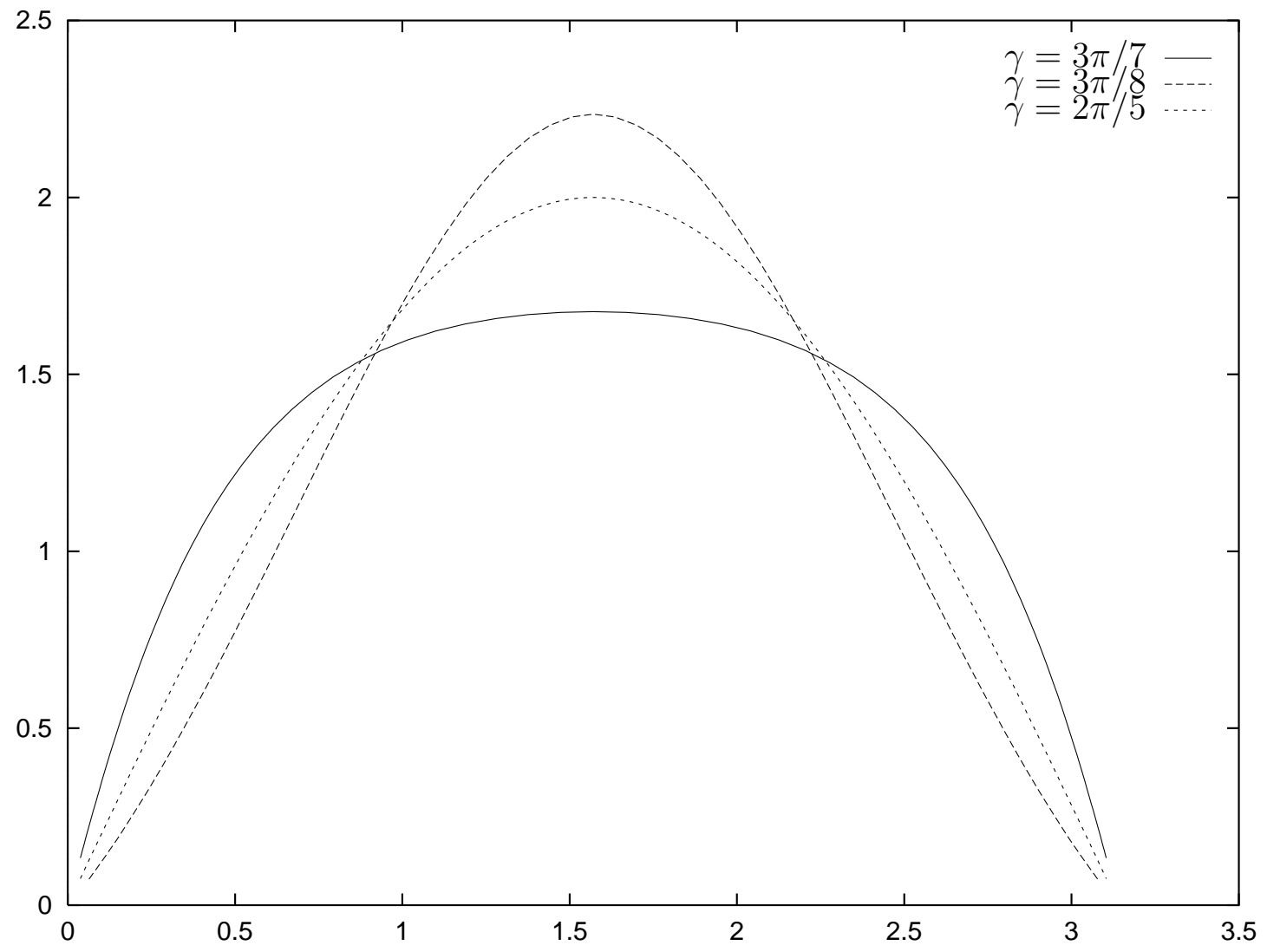

Figure 1. Dispersion relations for the holes in the $(1,+)$-string distributions in sector II for $\bar{c}=-5 \tilde{c}=1$ and different $\gamma$.

$$
\begin{aligned}
p_{h}^{1+} & =\frac{\pi}{2}+\frac{1}{2} p(\lambda, 0, \gamma)+2 \pi e_{1}(\lambda), \\
\varepsilon_{h}^{1+} & =\bar{c} \frac{1}{2} g(\lambda, 0, \gamma)+2 \pi \tilde{c} d_{1}(\lambda) .
\end{aligned}
$$

We have calculated the dispersion law numerically and show the results in figure 1. It is interesting to look closer at the point $\gamma=2 \pi / 5$, where the function $d_{1}(\lambda)$ simplifies and becomes $1 / 4 \pi g(\lambda, 0, \gamma)$, resulting in the simple dispersion law

$$
\varepsilon_{h}^{1+}=\frac{2 \pi}{\gamma} \frac{\bar{c}+\tilde{c}}{2} \sin p_{h}^{1+} .
$$

All dispersion laws possess a symmetry connected with the properties of the functions $p_{h}(\lambda)$ and $\varepsilon_{h}(\lambda)$ :

$$
\begin{aligned}
& p_{h}(\lambda)=p_{0}+\bar{p}(\lambda) \quad \text { and } \quad \bar{p}(-\lambda)=-p(\lambda), \\
& \varepsilon_{h}(\lambda)=\varepsilon_{h}(-\lambda) .
\end{aligned}
$$

One then derives easily

$$
\varepsilon_{h}\left(p_{h}\right)=\varepsilon_{h}\left(2 p_{0}-p_{h}\right) .
$$

In addition one has $0 \leq p_{h} \leq 2 p_{0}$. The constants $2 p_{0}$ are different, so in sector 0 they are $\pi / 2$ for both excitations, the same is true for the negative parity excitation in II. The other (positiv parity) excitation in II has $2 p_{0}=\pi$, while in IV one has $2 p_{0}=3 \pi / 2$. 
There is a general rule for the sum of those constants in every sector being $3 \pi / 2$ (due to antiferromagnetic ground state), but excitations containing more than one magnon, e.g. $(2,+)$-strings, must be taken with their appropriate multiplicity.

\section{The speed of sound in different sectors}

In this section we shall present some calculations for the speed of sound in sectors with one finite Fermi radius (the other one stays infinite) where this radius is either small or large, so that an approximation can be made. On the other hand, that gives just the values of the speed near to the border lines of the above mentioned sector which might be of interest for understanding the physics of the model. We shall limit our calculations to the case $\bar{c}>0, \tilde{c}<0$. Thus, the sectors in question are I and III. Sectors V and VI could be treated in an analogous way.

We start with sector I. The equations of the thermodynamic Bethe ansatz can be taken from [1] (equation (4.5)).

$$
\begin{aligned}
& \epsilon_{1+}(\lambda)=-2 \pi \bar{c} s_{1}(\lambda)-2 \pi \tilde{c} d_{1}(\lambda)+d_{1} * \epsilon_{1+}^{+}(\lambda), \\
& \epsilon_{1-}(\lambda)=2 \pi \tilde{c} s_{2}(\lambda)-s_{2} * \epsilon_{1+}^{+}(\lambda) .
\end{aligned}
$$

Here $\varepsilon_{i}(\lambda)$ are the dressed energies. Their lower index specifies the excitation, while the upper stands for the positive and negative parts respectively. The convolution is defined by

$$
a * b(\lambda)=\int_{-\infty}^{\infty} d \mu a(\lambda-\mu) b(\mu) .
$$

(For $s_{1}(\lambda)$ and $s_{2}(\lambda)$ see the appendix.)

Restricting $\gamma$ by $0<\gamma<2 \pi / 5$ we consider the case

$$
0<\beta=\bar{c}-\frac{|\tilde{c}|}{2 \cos \tilde{\gamma}} \ll 1
$$

with the usual definition $\tilde{\gamma}=\pi \gamma / 2(\pi-\gamma)$.

From equation (3.13) from [4] we know that $\beta=0$ describes the border line between sectors IV and I.

To employ the method of power expansions in the (small) Fermi radius $b$ of the $(1,+)$-strings, it is useful to reformulate equations (4.1) and (4.2) in the way

$$
\begin{aligned}
& \varepsilon_{1+}(\lambda)=-\bar{c} g(\lambda, \pi-2 \gamma)-\tilde{c} g(\lambda, \pi-3 \gamma)-\frac{1}{2 \pi} g(\lambda, \pi-3 \gamma) * \varepsilon_{1+}^{-}(\lambda), \\
& \varepsilon_{1-}(\lambda)=2 \pi \bar{c} s_{1}(\lambda)+\tilde{c} g(\lambda, \gamma)+\frac{1}{2 \pi} g(\lambda, \gamma) * \varepsilon_{1+}^{-}(\lambda) .
\end{aligned}
$$

Equation (4.5) determines the Fermi radius $b$, in lowest order the convolution term be neglected and $b$ is given by $\varepsilon_{1+}(b)=0$. The derivative $d \varepsilon / d p$ is calculated the way (see equation (5.1) in [1])

$$
\frac{d \varepsilon}{d p}=\frac{d \varepsilon}{d \lambda} \frac{d \lambda}{d p}=-\left.\frac{d \varepsilon}{d \lambda} \frac{2}{\varepsilon(\lambda)}\right|_{\bar{c}=\tilde{c}=1}
$$


The speed of sound is then given if $\lambda$ is put equal to $b$.

After rather long technical calculations we have obtained

$$
v_{1+}=\frac{4 \pi}{\pi-\gamma} \frac{1}{\sin \tilde{\gamma}} \sqrt{\frac{2 \cos \tilde{\gamma}-1}{2 \cos \tilde{\gamma}+1}}(\bar{c} \beta)^{1 / 2} .
$$

Equation (4.6) gives the same way

$$
v_{1-}=\frac{2 \pi}{\pi-\gamma}(2 \cos \tilde{\gamma}-1) \bar{c}+\frac{8}{\pi-\gamma} \sin \tilde{\gamma} \sqrt{\frac{2 \cos \tilde{\gamma}-1}{2 \cos \tilde{\gamma}+1}}(\bar{c} \beta)^{1 / 2} .
$$

Here the first term is just the limiting value on the border of sector IV.

Next we consider $\pi / 3<\gamma<2 \pi / 5$ and look for the border of sector I with sector II (see [1], equation (4.10)). It is given by

$$
0<\alpha=|\tilde{c}| \tan \left(\frac{\pi^{2}}{2 \gamma}\right)-\bar{c} \ll 1 .
$$

Then equation (4.1) is of Wiener-Hopf type and can be solved explicitely for large $b$ giving

$$
v_{1+}=\frac{2 \pi}{\gamma} \frac{3 \gamma-\pi}{\pi-2 \gamma} \frac{\alpha}{1+\tan \left(\frac{\pi^{2}}{2 \gamma}\right)} .
$$

From equation (4.2) one has to extract the asymptotics of $\varepsilon_{1-}(\lambda)$ for large $\lambda$ (Fermi radius is infinite). The first term on the RHS gives the limiting value of $v_{1-}$ on the border of sector II (it is non-vanishing). Remarkably for $\alpha>0$ the asymptotics is always given by the second term with the convolution. So the result is of the same order in $\alpha$ as $v_{1+}$ :

$$
v_{1-}=\frac{2 \pi}{\gamma} \frac{3 \gamma-\pi}{\gamma} \frac{\alpha}{1+\tan \left(\frac{\pi^{2}}{2 \gamma}\right)} .
$$

Therefore $v_{1-}$ shows a discontinuity on the border I/II.

After sector I we consider sector III. Then one has $2 \pi / 5<\gamma<\pi / 2$. We start again with the border to sector IV.

$$
0<\beta^{\prime}=\bar{c}-2 \cos \tilde{\gamma} \tilde{c} \ll 1 .
$$

The Fermi radius is determined again from equation (4.5), but now instead of power expansion we have to solve a Wiener-Hopf problem due to the fact that $b$ is large. The calculation is straightforward after realizing that for the positive frequency part of the inhomogenous function one has to take into account the dominant two poles (instead of one). As already mentioned in [1] the fact that filling of the $(1,+)$-strings starts at infinity causes the appearance of two velocities for them taken at $\lambda=b$ and $\lambda=\infty$. While the calculations for both are different the results coincide in first order.

The same happens for $v_{1-}$ calculated from the asymptotics of equation (4.6). We remind the reader that $v_{1-}$ vanishes at the border. Finally we have obtained

$$
v_{1+}^{(1)}=v_{1+}^{(2)}=v_{1-}=\frac{2 \pi}{\pi-\gamma} \frac{\beta^{\prime}}{1+2 \cos \tilde{\gamma}} .
$$


We have no explanation for that striking fact, that all velocities are equal in first order. Nevertheless we are sure that the effect does not survive in higher order.

To close that part we have to consider the border line with sector II (see [1], equation $(4.8))$.

$$
0<\alpha^{\prime}=|\tilde{c}| 2 \gamma d_{1}(0)-\bar{c} \ll 1 .
$$

The Fermi radius $b$ is determined by equation (4.1) using power expansion. The calculation is straightforward, but rather tedious because of the presence of the function $d_{1}(\lambda)$. But the order of magnitude can be estimated easily. The two velocities $v_{1-}^{(1)}$ and

$v_{1+}^{(2)}$ (taken at $\lambda=\infty$ ) obtain corrections to their (constant) limiting values from sector II of the order $\sqrt{\alpha^{\prime}}$. The third velocity $v_{1+}^{(1)}$ (taken at $\lambda=b$ ) is of order $\sqrt{\alpha^{\prime}}$.

We did not consider the border of sectors I and 0 for $0<\gamma \leq \pi / 3$ because both sectors are separated form each other by a singular line with a highly degenerate ground state which is different from the other lines considered above.

\section{Conclusions}

First we make some general comments on the character of the behaviour of the velocities considered. We parametrize the coupling constants by two parameters, one $c$, setting the energy scale (below put to one), the other one an angle $\varphi$, which describes their ratio.

$$
\begin{aligned}
\bar{c} & =c \sin \varphi, \\
\tilde{c} & =c \cos \varphi .
\end{aligned}
$$

In general from our results in section 4 one can see that the behaviour near the sector borders is either linear or a square root in cosine/sine-functions of $\varphi$. The fact which one is chosen is determined by the radius $b$. Large $b$ produces Wiener-Hopf equations and linear behaviour, small $b$ causes power expansions and a square root behaviour. Considering sector I only, one could superficially conclude that this depends only on the sector on the other side of the border. Our analysis in sector III shows that the situation reverses with respect to sector I.

In just one case we have found a discontinuity of one of the velocities when a sector border is passed (see equation (4.12)). It is the case for $v_{1-}$ on the border I/II. Nothing of this kind happens for sector III, because for small $\alpha^{\prime}$ we have power expansion, noncapable of producing such an effect. On the other hand (for small $\beta^{\prime}$ ) $v_{1-}$ vanishes on the other side of the border in sector IV. In general, one would expect a discontinuity only when a singular line is crossed which means that an excitation with infiniteFermi zone disappears at once (see below). It is rather instructive to compile all analytically calculated velocities in one picture at the special point $\gamma=2 \pi / 5$ which we have done in figure 2 .

For convenience, we have given the analytical shape in the various sectors in table 2. Looking at figure 2 one clearly sees two effects. First the two singularity lines at $\varphi=\pi / 2$ (the vanishing of the $(2,+)$-strings) and at $\varphi=3 \pi / 4$ (the vanishing of the 


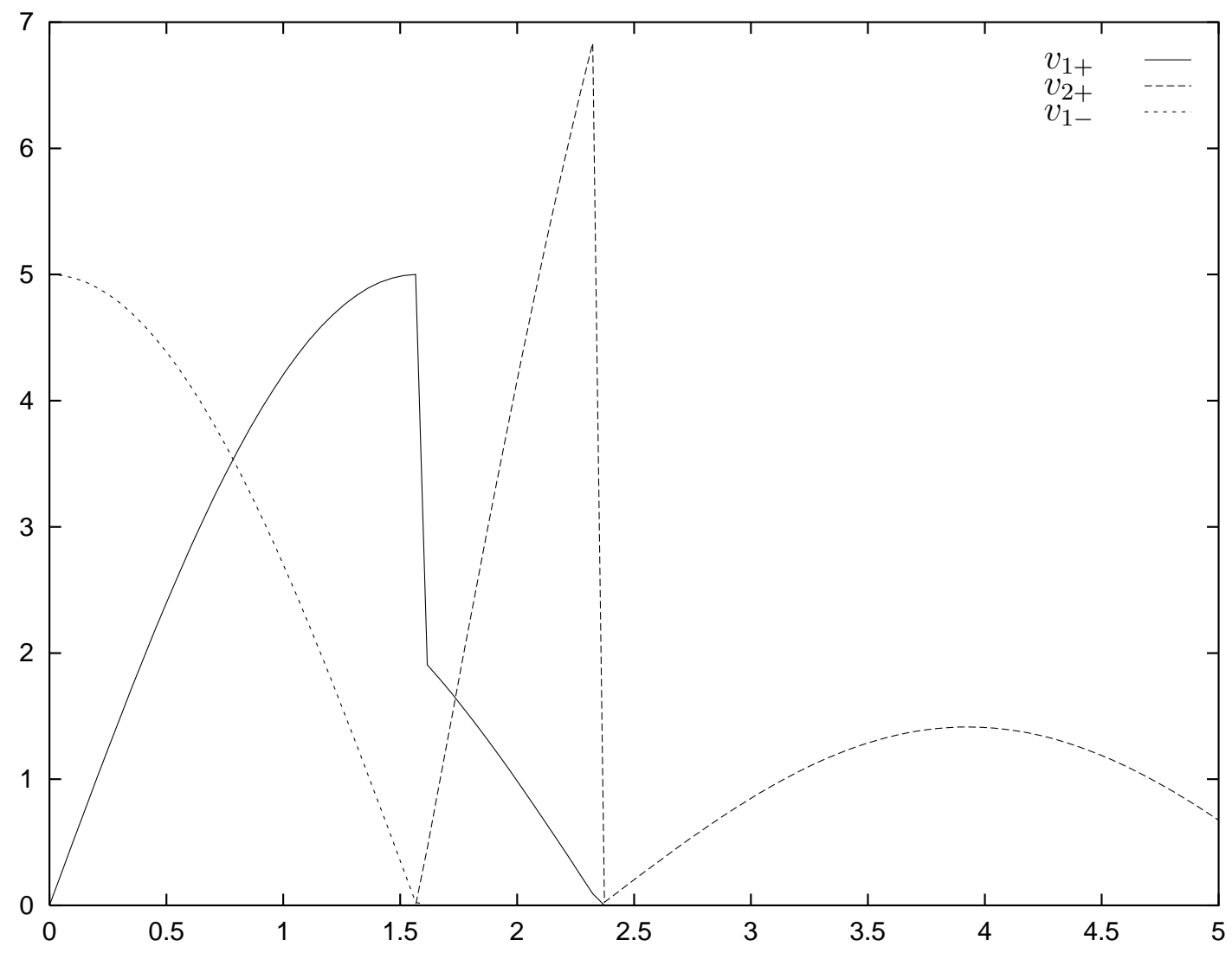

Figure 2. The speeds of sound as functions of the angle $\varphi$ for $\gamma=2 \pi / 5$ and those sectors where all speeds of sound are known.

\begin{tabular}{|c||c||c||c||c|}
\hline sectors & 0 & II & IV & V \\
\hline borders & $0, \pi / 2$ & $\pi / 2,3 \pi / 4$ & $3 \pi / 4,2 \pi-\arctan 4$ & $2 \pi-\arctan 4,2 \pi$ \\
\hline$v_{1+}$ & $5 \sin \varphi$ & $5 / 2 \sin \varphi+5 / 2 \cos \varphi$ & 0 & 0 \\
\hline$v_{2+}$ & $5 \cos \varphi$ & 0 & 0 & \\
\hline$v_{1-}$ & 0 & $-10 \cos \varphi$ & $-5 / 3 \sin \varphi-5 / 3 \cos \varphi$ & \\
\hline
\end{tabular}

Table 2. The speeds of sound and the sector borders as functions of the angle $\varphi$ for $\gamma=2 \pi / 5$.

$(1,+)$-strings) cause discontinuities for the speed of the remaining excitation. While the first line is universal for arbitrary $\gamma$, the second one is peculiar to $\gamma=2 \pi / 5$. For other $\gamma$ that line is replaced by sectors I or III.

Second one sees the two conformal points where two velocities intersect, one at $\varphi=\pi / 4$ and the other at $\varphi=\pi-\arctan 5$ [4].

Our calculations in the previous section allow now to look closer at what happens for $\pi / 3<\gamma<2 \pi / 5$ when the above mentioned singularity line is replaced by sector I. As far as the limits of sectors II and IV are concerned the only change is that $v_{1-}$ no longer vanishes at the border of sector IV. Now we make use of equations (4.9), (4.10), (4.11) and (4.12). They tell us first that both velocities must reach their maximum (at different 
$\varphi$ ) within sector I and second that there is (at least one) point of intersection in the same sector. In that point the finite size corrections must exhibit a rather simple structure like in [6].

We conjecture that there is no such effect in sector III but a strict prove requires further calculations. In addition, our calculations show another interesting effect, at $\beta^{\prime}=0$ all velocities vanish.

Finally we remark that we expect the theory at $\gamma=2 \pi / 5$ and both velocities equal to be highly symmetric and of particular interest. Up to now it is only known that its central charge is 2 . We were not able to calculate the operator dimensions analytically, because contrary to what happened in case of paper [5] it requires the knowledge of the dressed charge [6] and hence the explicit factorization of the kernel.

\section{Acknowledgment}

The authors would like to thank H. Frahm for helpful discussions.

\section{Appendix}

$$
\begin{aligned}
& \left.s_{1}(\lambda)=\frac{1}{2 \gamma \cosh (\pi \lambda / \gamma}\right) \\
& s_{2}(\lambda)=\frac{1}{2(\pi-2 \gamma) \cosh (\pi \lambda /(\pi-2 \gamma))} \\
& g(\lambda, \alpha)=\int_{-\infty}^{\infty} e^{i \omega \lambda} \frac{\cosh \omega \alpha / 2}{\cosh \omega(\pi-\gamma) / 2} d \omega
\end{aligned}
$$

\section{References}

[1] Dörfel B - D and Meißner St 1998 J. Phys. A: Math. Gen. 3161

[2] de Vega H J and Woynarovich F 1992 J. Phys. A: Math. Gen. 254499

[3] Meißner St and Dörfel B - D 1996 J. Phys. A: Math. Gen. 291949

[4] Dörfel B - D and Meißner St 1996 J. Phys. A: Math. Gen. 296471

[5] Dörfel B - D and Meißner St 1997 J. Phys. A: Math. Gen. 301831

[6] Woynarovich F 1989 J. Phys. A: Math. Gen. 224243 


\section{Figure and table captions}

Figure 1. Dispersion relations for the holes in the $(1,+)$-string distributions in sector II for $\bar{c}=-5 \tilde{c}=1$ and different $\gamma$.

Figure 2. The speeds of sound as functions of the angle $\varphi$ for $\gamma=2 \pi / 5$.

Table 1. All sectors appearing in the phase diagram. Upper indices indicate infinite and finite Fermi zones. In the latter case, the second index distinguishes, wether the filling starts at $\lambda=0$ or $\lambda=\infty$.

Table 2. The speeds of sound and the sector borders as functions of the angle $\varphi$ for $\gamma=2 \pi / 5$. 